artículos derivados de proyectos de investigación 

CUAD. CONTAB. / BOGOTÁ, COLOMBIA, 16 (42): 553-578 / SEPTIEMBRE-DICIEMBRE 2015 / 553

\section{E-control en las entidades de la rama ejecutiva del orden nacional en Colombia*}

doi:10.11144/Javeriana.cc16-42.eere

\section{Raúl Leandro Gutiérrez-Ruiz}

Contador público, Universidad de La Salle. Miembro del semillero de investigación Responsabilidad, rendición de cuentas y transparencia.

Correo electrónico: rgutierrez42@unisalle.edu.co

\section{Diego Fernando Católico-Segura}

Contador público, Universidad Nacional de Colombia.

Magíster en contabilidad y finanzas, Universidad de

Zaragoza, España. Docente investigador, Universidad

de La Salle. Miembro del grupo de investigación

Responsabilidad, rendición de cuentas y transparencia.

Correo electrónico: dfcatolico@unisalle.edu.co

\footnotetext{
* Artículo derivado de un proceso de investigación que se adelantó bajo la modalidad de grado producción intelectual relevante, avalada por el Programa de Contaduría Pública de la Universidad de La Salle. El título de la propuesta de la modalidad de grado fue "E-control en las entidades de la rama ejecutiva del orden nacional en Colombia". La fecha de inicio del proceso fue julio de 2014 y la fecha de terminación julio de 2015.
} 
Resumen La corrupción es uno de los flagelos más concurrentes en el mundo y Colombia no ha sido ajena a él, pues de acuerdo con las mediciones realizadas por Transparencia Internacional (2012-2014), el índice de percepción de corrupción en el país es significativo. Para minimizar esta situación, se han implementado varias medidas de control y sanción, como la obligación de hacer visible el informe de control interno en los sitios web de las entidades estatales, para posibilitar el control social, según lo dispuesto en la Ley 1474 de 2011. Así, la presente investigación aborda de forma empírica y descriptiva el grado de cumplimiento en la publicación de ese informe por parte de las entidades de la rama ejecutiva del orden nacional del nivel central en Colombia, para analizar su contenido y poder entender qué tanto contribuyen al control social. Los resultados evidencian grados intermedios y bajos de cumplimiento tanto en la publicación de los informes, como en el contenido que se espera que tengan, lo cual limita el acceso a una información útil para realizar control social sobre este tipo de entidades.

Palabras clave Control interno; control social; informe pormenorizado de control interno; internet.

\section{Código JEL M42}

\section{E-Control in the National Executive Branch in Colombia}

\footnotetext{
Abstract Corruption is one of the most concurrent scourges in the world, and Colombia has not been a stranger to it. According to the measurements performed by International Transparency (2012 - 2014) there is a significant corruption perception index in the country. In order to minimize this situation several control and penalty measures have been put into place, such as the obligation to make public the internal control report at the websites of state institutions, as set forth in Law 1474, 2011. So, this research tackles in an empiric and descriptive way the level of compliance in the publishing of said report by institu-
}

tions of the executive branch at the central national level in Colombia, in order to analyze its content and be able to understand how much they contribute to social control. Results show low and intermediate levels of compliance both in the publishing of the results and in the expected contents they should have, which limits the access to useful information for the purposes of the social control to be performed on these institutions.

Keywords internal control; social control; detailed internal control report; internet.

\section{E-controlo nas instituições da rama executiva da ordem nacional na Colômbia}

Resumo A corrupção é um dos flagelos mais concorrentes no mundo e a Colômbia não foi alheia a ela, pois segundo as medições feitas por Transparência Internacional (2012-2014), o índice de percepção de corrupção no país é significativo. Para minimizar esta situação, implementaram-se várias medidas de controle e sanção, tais como a obrigação de visibilizar o relatório de controle interno nos sites das instituições estatais, para possibilitar o controle social, segundo o disposto na Lei 1474 de 2011. Assim, a presente pesquisa aborda de forma empírica e descritiva o grau de cumprimento na publicação desse relatório por parte das entidades da rama executiva da ordem nacional do nível central na Colômbia, para analisar seu conteúdo e conseguir entender quanto contribuem para o controlo social. Os resultados evidenciam graus intermédios e baixos de cumprimento tanto na publicação dos relatórios quanto no conteúdo que eles devem ter, o que limita o acesso a uma informação útil para realizar controlo social sobre este tipo de entidades.

Palavras-chave Controlo interno; controlo social; relatório pormenorizado de controlo interno; internet. 
E-CONTROL EN LAS ENTIDAdES dE LA RAMA EJECUTIVA / R. GutiÉRREZ, D. CATólico-segura / 555

\section{Introducción}

De acuerdo con el informe sobre el índice de percepción de la corrupción realizado por Transparencia Internacional (2014), más de dos tercios de los 175 países incluidos en el Índice de 2014 alcanzan niveles significativos de percepción sobre la existencia de escenarios de corrupción, consecuencia de la creciente evidencia de los casos de fraude y corrupción tanto en el ámbito estatal, como en el privado.

Según este informe, Colombia ocupa el puesto 94 con una puntuación de 37, la misma que obtuvo en informes similares desarrollados para 2013 y 2012. Esto quiere decir que Colombia tiene escenarios de corrupción recurrente en sus instituciones y empresas.

Esto no es nuevo, pues Colombia ha sido un país con problemas sociopolíticos desde hace más de 50 años, cuya imagen ha sido cuestionada a raíz del surgimiento de fuerzas armadas revolucionarias, el auge del narcotráfico y la presencia de los delitos de cuello blanco en las más importantes instituciones y ciudades del país. Esto último se configura en prácticas de corrupción, como lo ha expuesto la Oficina de Naciones Unidas contra la Droga y el Delito, UNODC (2015):

El concepto de corrupción es amplio. Incluye soborno, fraude, apropiación indebida $u$ otras formas de desviación de recursos por un funcionario público, pero no es limitado a ello. La corrupción también puede ocurrir en los casos de nepotismo, extorsión, tráfico de influencias, uso indebido de información privilegiada para fines personales y la com- pra y venta de las decisiones judiciales, entre varias otras prácticas (p. 1).

Hoy, este problema de delincuencia - la corrupción-es el flagelo que más noticias abarca y preocupa a la sociedad colombiana, y el que tenía a las entidades públicas y privadas en un nivel de desconfianza (percepción de corrupción) del 81\% en 2011, según el Latin American Public Opinion Project, LAPOP, y el Barómetro de las Américas 2011 (Rodríguez \& Seligson, 2011).

Lo anterior tiene mayor relevancia cuando se enumeran los casos de corrupción que se han presentado en las entidades públicas y con mayor incidencia en las que integran la rama ejecutiva del orden nacional. Casos como los de Agro Ingreso Seguro en el Ministerio de Agricultura y Desarrollo Social, el desvío de recursos parafiscales de la salud en el Ministerio de Salud y el caso de las chuzadas del antiguo Departamento Administrativo de Seguridad (DAS), entre otros, son evidencia de las situaciones vividas en los últimos años, con importantes consecuencias económicas, políticas y sociales.

A partir de los efectos de la corrupción y con el ánimo de evitar estas situaciones, se observa la necesidad de afianzar los controles y la supervisión, tanto los que se desarrollan dentro de las entidades, como aquellos que se pueden realizar externamente, como el control social. En este sentido, el gobierno nacional ha realizado ajustes continuos en el Modelo Estándar de Control Interno, en las exigencias de rendición de cuentas de las entidades y en la transparencia informativa sobre los asuntos relevantes de la entidad. Un ejemplo de lo anterior 
ha sido la promulgación de la Ley 1474 de 2011, más conocida como Estatuto Anticorrupción, la cual ha establecido un conjunto de medidas orientadas a fortalecer los mecanismos de prevención, investigación y sanción de actos de corrupción, con el fin lograr una mayor efectividad en el control de la gestión pública para mitigar los riesgos de corrupción.

En este sentido, esta ley señala como medida de prevención la publicación periódica del informe de control interno en los sitios web de las entidades del Estado, lo cual es responsabilidad del jefe de cada unidad de control interno de las entidades que hacen parte de la rama ejecutiva del orden nacional y territorial.

Esta medida se sintoniza con la estrategia de gobierno en línea (e-government) que el gobierno nacional ha venido implementando para posibilitar escenarios de acceso a la información y mejora en la prestación de los servicios (CONPES, 2010a), con mecanismos basados en las tecnologías de la información y la comunicación (TIC), por lo que al posibilitar la publicación del informe de control interno en los sitios web contribuye a mejorar la comunicación entre la entidad y el control que ejerce la sociedad sobre las instituciones, lo que podría configurar una práctica del e-control en las instituciones del Estado colombiano.

De este modo, la presente investigación indaga sobre el grado de cumplimiento en la publicación del informe de control interno en las páginas web de las entidades del Estado y analiza su contenido, para evaluar qué tanto favorecen el control social que se pueda ejercer en estas entidades. Para ello, este artículo inicia con la presentación de los conceptos y lineamientos que enmarcan el tema a tratar, y luego desarrolla el trabajo empírico sobre los informes expuestos en los sitios web de las entidades, considerando la metodología propuesta para el estudio, los resultados obtenidos y las conclusiones alcanzadas al respecto, como se muestra a continuación.

\section{El control interno y la participación ciudadana}

El término control, en su acepción más extendida, hace referencia a "comprobación, inspección, fiscalización o intervención”, es decir, consiste en contrastar si unas actuaciones están o no en consonancia con normas o criterios dados que marcan cómo se debe hacer; todos estos conceptos son aplicables a la gestión de la actividad económica-financiera (López-Hernández \& Cabeza del Salvador, 2002).

La función de control se encarga a órganos formal y materialmente administrativos, y consiste en el uso de medios jurídicos para anular, rectificar o sancionar la gestión pública ilegal o ineficaz, con medios técnicos denominados "procedimientos administrativos", que constituyen controles propiamente dichos, auditorías y procesos para determinar la legalidad de los actos de las autoridades administrativas (Márquez, 2010).

Por su parte, el Committee of Sponsoring Organizations of the Treadway Commission, COSO (2013) define el control interno como un proceso efectuado por la junta de administración, gerencia y otro personal de la entidad, diseñado para proporcionar una seguridad razonable en cuanto a la consecución de los objetivos relacionados con las operaciones, la 
elaboración de informes y el cumplimiento (p. 3), el cual es aplicado indistintamente en todas las organizaciones y en especial en los países que tienen un alto desarrollo gerencial (Montilla, Montes \& Mejía, 2007).

Por ello, al ser el control interno una noción universal, se entiende y se hace necesaria una aplicación en lo que compete tanto a lo privado como a lo estatal, por cuanto los dos sectores tienen la necesidad de generar confianza y aseguramiento en las políticas, decisiones y administración de los recursos.

Así ha sido entendido en el ámbito global. De allí surgen entidades como el Consejo de Normas Internacionales de Auditoría y Aseguramiento (IAASB, por sus siglas en inglés), así como los modelos de control interno público establecidos en cada país, por ejemplo, el Committee of Sponsoring Organizations of the Treadway Commission (COSO), en Estados Unidos; el Criteria of Control Board (CoCo), en Canadá; el Australian Control Criteria (ACC), en Australia y el Tribunal de Cuentas en España (Montilla, Montes \& Mejía, 2007).

Colombia no ha sido la excepción en establecer su modelo para el sector estatal, pues por medio de la Ley 87 de 1993 y los Decretos 1599 de 2005 y 943 de 2014, ha determinado la definición y la metodología a seguir con base en el Modelo Estándar de Control Interno (MECI), para ordenar el diseño, la operación y el seguimiento del sistema en entidades del Estado.

El MECI también busca unificar la estructura y criterios básicos de control interno para las entidades del Estado, al integrar los conceptos de control, con el fin de aclarar el marco con- ceptual existente. Por ello, el propósito esencial del MECI es orientar a las entidades hacia el cumplimiento de sus objetivos y la contribución de estos a los fines esenciales del Estado (DAFP, Dirección de Políticas de Control Interno y Racionalización de Trámites, 2008), para lo cual se estructura en tres grandes módulos, desagregados en componentes y elementos con sus respectivos productos mínimos de control:

- Módulo de Control de Planeación y Gestión: este módulo agrupa los parámetros de control que orientan la entidad hacia el cumplimiento de su visión, misión, objetivos, principios, metas y políticas, y los aspectos que permiten el desarrollo de la gestión, entre los que están: talento humano, planes, programas, procesos, indicadores, procedimientos, recursos y administración de los riesgos.

\section{- Módulo de Control de Evaluación y Se-} guimiento: agrupa los parámetros que garantizan la valoración permanente de los resultados de la entidad, mediante sus diferentes mecanismos de verificación, evaluación y seguimiento.

\section{- Módulo de Información y Comunicación:} tiene una dimensión estratégica fundamental por cuanto vincula a la entidad con su entorno y facilita la ejecución de sus operaciones internas, al darle al usuario una participación directa en el logro de los objetivos. Es un factor determinante, siempre y cuando se involucre en todos los niveles y procesos de la organización.

Con este módulo se espera mejorar la transparencia frente a la ciudadanía por medio de la rendición de cuentas a la comunidad 
y el cumplimiento de las obligaciones frente a las necesidades de los usuarios (DAFP, 2014b).

De acuerdo con la estructura señalada, el Departamento Administrativo de la Función Pública, DAFP, como líder de la política de control interno, establece guías que permiten a las oficinas de control interno de las entidades del Estado realizar su labor de auditoría interna.
Esta metodología se estructura principalmente con base en las normas internacionales para el ejercicio profesional de auditoría interna (DAFP \& Instituto de Auditores Internos de Colombia, 2013).

En 2013, el DAFP publicó la Guía de auditoría para entidades públicas, que establece una propuesta metodológica para ejecutar el proceso de auditoría interna, el cual se define en las siguientes fases:

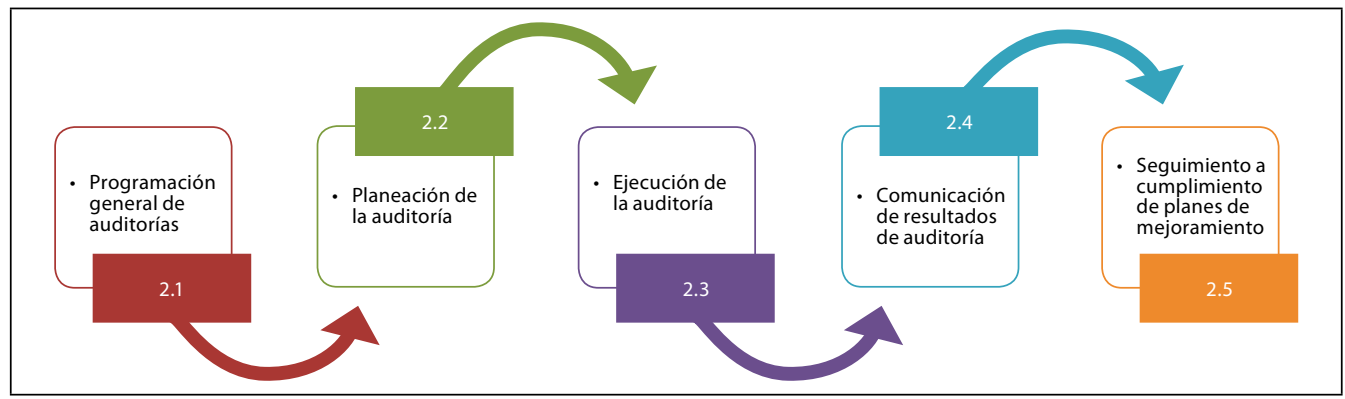

Figura 1

Fases para el proceso de auditoría

Fuente: Guía de auditoría para entidades públicas (DAFP \& Instituto de Auditores Internos de Colombia, 2013, p. 24)

Como se puede observar en la figura 1 , el trabajo de auditoría interna en las entidades públicas contiene casi los mismos lineamientos de una auditoría en general, como establecen las Normas Internacionales de Auditoría (IAS, por sus siglas en inglés) y con énfasis en las Normas Internacionales para el Ejercicio Profesional de la Auditoría Interna (en adelante, NIEPAI). En teoría, la fase 4 resume las anteriores etapas, ya que presenta los resultados de la auditoría y en ella se suscriben los planes de acción o mejoramiento, es decir, el contenido que debería llevar el informe de Auditoría Interna (DAFP \& Instituto de Auditores Internos de Colombia, 2013).
En consecuencia de lo anterior, la auditoría interna, por medio del informe, busca dar cumplimiento al principio de publicidad establecido por la Carta Política, la cual, de conformidad con lo preceptuado en su artículo 209, obliga a la administración a poner en conocimiento de sus destinatarios los actos administrativos (Corte Constitucional, 1999b).

Esto es concordante con uno de los objetivos del MECI (teniendo en cuenta que en la Evaluación del Sistema de Control Interno, SCI, es necesaria la emisión de un informe rindiendo cuentas sobre el estado del SCI), al preceptuar que la Oficina de Control Interno 
—o quien haga sus veces_- deberá presentar informes a la dirección y al comité de coordinación de control interno de la entidad, haciendo énfasis en la existencia, funcionamiento y coherencia de los componentes y elementos que lo conforman, con el propósito de que allí se evalúen, decidan y adopten oportunamente las propuestas de mejoramiento del Sistema (DAFP, Dirección de Políticas de Control Interno y Racionalización de Trámites, 2008).

En este orden de ideas, el informe constituye una herramienta de comunicación fundamental entre la entidad y la sociedad, más aún cuando el gobierno nacional ha entendido la necesidad de hacerlo público en los sitios web de las entidades, como lo señaló el artículo 9 de la Ley 1474 de 2011, al establecer su divulgación periódica (cada cuatro meses). Con ello, se contribuye al control social que ejercen distintos estamentos y se fortalece la estrategia de gobierno en línea y de rendición de cuentas establecida en los documentos Conpes 3650 y 3654 de 2010, y en la directiva presidencial 002 de 2000, reglamentada por los Decretos 1151 de 2008 y 2693 de 2012, y la Ley 1712 de 2014, Ley de transparencia y del derecho de acceso a la información pública nacional, todas estas enmarcadas en derechos constitucionales como los establecidos en los artículos 20, 23, 74 y 209 de la Carta Política nacional.

La divulgación de este informe a la ciudadanía en general y no solo a los supervisores o entes de control de las entidades es determinante en el ejercicio del control social, como lo establece el artículo 2 de la Carta Política Colombiana, al facilitar la participación de todos en las decisiones que los afectan y en la vida económi- ca, política, administrativa y cultural de la Nación (Colombia, Constitución Política, 1991).

Lo anterior es una práctica necesaria y que toda persona que haga parte de un Estado debería ejercer, si se tiene en cuenta que los ciudadanos están más alejados de las administraciones públicas que, por ejemplo, los accionistas de una empresa privada, por lo que el informe del auditor es necesario para analizar la fiabilidad de la información presentada y poder evaluar el estado de la entidad y la gestión llevada a cabo (Pucheta-Martínez \& CortésFleixas, 2005).

La participación ciudadana, como parte del control social, corresponde a un escenario propio de las democracias. Por eso, en la Directiva Presidencial número 12 de 2002, el gobierno nacional fijó algunos lineamientos orientados hacia la lucha contra la corrupción, al integrar a la ciudadanía por medio de las veedurías, al continuo seguimiento en la contratación y manejo de los recursos públicos.

Así mismo, la emisión de ese informe hace parte de la lógica de rendición de cuentas, es decir, hace parte del proceso proactivo mediante el cual los agentes públicos informan y explican sus acciones a otro(s) que tienen el derecho de exigirlo, debido a la presencia de una relación de poder y a la posibilidad de imponer algún tipo de sanción por un comportamiento inadecuado o de premiar un comportamiento destacado (CONPES, 2010b), por lo que se ha convertido en un factor clave de los estados y de la sociedad a la hora de administrar y controlar las acciones y recursos entregados a entidades y funcionarios para su ejecución. Esto no solo permite una mayor eficacia en la ejecución 
y administración de recursos, sino también mayor confianza ciudadana.

Por otra parte, facilitar tal información en los sitios web busca un medio de comunicación de mayor cobertura, acceso y oportunidad, lo que se adhiere a las prácticas de e-government o gobierno en línea que el Estado ha venido adelantando y, en particular, que las entidades gocen de una transparencia informativa online, con lo cual se configura una práctica del e-control, es decir, de posibilitar un escenario de control haciendo uso de las tecnologías de la información y la comunicación, pues el primer paso se da con la exposición de los resultados de los ejercicios de auditoría para ponerlos al alcance de todos los interesados.

De allí la importancia de estos conceptos y políticas traídas al contexto colombiano por el gobierno, para estructurar una metodología de control y vigilancia en las entidades gubernamentales, al dar acceso a una información que resulta relevante para conocer lo adecuado de las actuaciones y gestiones que llevan a cabo las entidades del Estado; por ello, la relevancia de la presente investigación para evidenciar las prácticas en esta materia.

\section{Metodología del estudio}

La investigación que se adelanta es empíricodescriptiva, toda vez que evalúa el grado de cumplimiento en la publicación de los informes pormenorizados de control interno de las entidades de la rama ejecutiva del orden nacional del nivel central en sus sitios web, de acuerdo con lo preceptuado en el Estatuto Anticorrupción.
De este mismo modo, se analiza el contenido que se consigna en esos informes siguiendo los lineamientos trazados por el DAFP (2014a) para tal fin, revisando no solo el cumplimiento del contenido, sino también las descripciones que se hacen en cuanto a los avances y dificultades que se estén presentando para que así las partes interesadas puedan realizar un mejor control social.

A partir de lo anterior, las hipótesis de trabajo se soportan en los siguientes enunciados:

\begin{abstract}
$\mathrm{H}_{1}$ : Las entidades de la rama ejecutiva del orden nacional nivel central logran un cumplimiento total en la publicación de los informes pormenorizados que contienen el estado del control interno, en sus sitios web. $\mathrm{H}_{2}$ : Los informes cumplen los requerimientos de información mínima a tratar en estos y esa información es la que se espera sea divulgada a las partes interesadas.
\end{abstract}

La primera hipótesis se sustenta en el amparo de la Carta Política colombiana, cuyo artículo 74 da a todo ciudadano el legítimo derecho de acceso a la información pública, garantiza la participación democrática y el ejercicio de los derechos políticos, que cumplen una función instrumental para la efectividad de otros derechos constitucionales y contribuye a la transparencia de la gestión pública, de manera que configura un mecanismo de control ciudadano de la actividad estatal (Corte Constitucional, 2013).

Adicionalmente, el cumplimiento total se ampara en la Ley 1474 de 2011, por medio de la cual se creó el requerimiento de divulgación de 
este tipo de informes, pues allí se establece una sanción al jefe de la oficina de control interno si no se cumple este mandato (falta disciplinaria grave).

La segunda hipótesis tiene sentido en la medida en que el informe constituye la fase final del proceso de auditoría y en el mismo se recogen todos los hallazgos detectados y el soporte documental para sustentar el dictamen emitido (DAFP \& Instituto de Auditores Internos de Colombia, 2013).

De igual forma, la evaluación sobre el estado de control interno debe entenderse realizada a todos los lineamientos dados en la estructura del MECI, es decir, llegando por lo menos hasta los "productos mínimos", aquellos considerados por el consejo asesor del gobierno nacional, como los mínimos necesarios para que el sistema de control interno esté en un nivel satisfactorio (DAFP, 2014b).

Si bien el Manual Técnico del Modelo Estándar de Control Interno para el Estado colombiano, MECI 2014, se limita al mero cumplimiento de informar si cumplen o no con el contenido que debe tener el producto mínimo, el DAFP estableció por medio del modelo de informe que se debe dar cuenta de los avances, dificultades y recomendaciones en cada uno de los productos mínimos a informar.

El mandato legal para la presentación de los informes objetos de evaluación, rige únicamente para la rama ejecutiva de orden nacional o territorial; la primera es la de mayor trascendencia en el Estado, pues las entidades de este tipo son las que mantienen la unidad nacional del país y no poseen una autonomía administrativa y financiera, por lo cual dependen de la autoridad suprema (Católico, Gómez \& Gómez, 2014).

Teniendo en cuenta lo anterior, esta investigación indagará en las 45 entidades pertenecientes a la rama ejecutiva del orden nacional nivel central, compuesta por la presidencia y la vicepresidencia de la república, dieciséis (16) ministerios, ocho (8) departamentos administrativos, una (1) superintendencia, diez (10) consejos superiores y ocho (8) unidades administrativas especiales (Católico, Gómez \& Gómez, 2014). La muestra real de entidades a evaluar será de 29 , toda vez que no todas cuentan con sitio web y para el caso de la vicepresidencia, se analizará en conjunto con la presidencia.

Sobre este conjunto de instituciones se realiza una evaluación respecto al grado de cumplimiento de la publicación en su sitio web de los informes cuatrimestrales pormenorizados de control interno, de acuerdo con lo establecido en el Estatuto Anticorrupción (Ley 1474 de 2011).

Así mismo, se evalúa la información allí contenida, siguiendo los requerimientos del Manual Técnico del MECI (2014), en cuanto a los módulos de información, que comprenden: tres componentes de planeación y gestión desarrollados en 10 elementos (33 productos mínimos); tres componentes de evaluación y seguimiento desarrollados en tres elementos (7 productos mínimos); y un componente de eje transversal sobre información y comunicación desarrollado en dos elementos (9 productos mínimos).

La anterior descripción de la información a reportar empezó a regir paulatinamente para 
todas las entidades a partir de mayo de 2014 (fecha en la cual se actualizó el MECI, incorporando nuevos productos mínimos de información), siendo noviembre y diciembre de 2014 los meses de culminación para la implementación del nuevo modelo, razón por la cual algunas entidades no presentaron toda la información requerida, ya que no culminaron su proceso de adopción. Esto se debe a que la evaluación que se realiza sobre el contenido de los informes se hace con referencia a aquellos que fueron publicados en el corte a noviembre de 2014, que en total fueron 25.

Para evaluar los indicadores antes mencionados, cada uno de ellos se trata como una variable dicotómica, es decir, asume una puntuación entre 0 y 1 , siendo "cero" cuando no cuenta con la divulgación del producto mínimo y "uno" cuando cuenta con la información. La misma escala de puntuación se da para medir el cumplimiento de publicación del informe en el sitio web, donde "cero" es cuando no cuenta con la publicación del informe, y "uno" cuando el informe fue publicado.

De la evaluación realizada se obtiene una puntuación global, la cual permite determinar el grado de cumplimiento en la divulgación de los informes y del contenido de los productos mínimos en cada uno de los informes analizados.

Con lo anterior, se realiza un ranking de $e$ control de las entidades que dan cuenta de los productos mínimos de acuerdo con los parámetros establecidos, para mostrar el grado de apropiación de las entidades del Estado de los elementos y de los productos consignados en el Modelo Estándar de Control Interno y, por ende, reconocer la presentación completa de la información que se reporta en el informe de control interno para mitigar riesgos de corrupción y proporcionar a la ciudadanía instrumentos de control que posibiliten la actuación y/o toma decisiones sobre estos.

\section{Resultados del estudio}

\section{Hipótesis de trabajo 1}

Para la resolución de la primera hipótesis de trabajo, se realizó una indagación en los sitios web de las entidades estatales objeto de estudio, teniendo como referencia los 10 informes que debieron emitir las entidades del Estado, que corresponden a los períodos desde la promulgación de la Ley 1474 de 2011 (Estatuto Anticorrupción) hasta noviembre de 2014. La indagación de los informes en los sitios web se realizó durante diciembre de 2014 y enero de 2015.

A continuación, se presentan los resultados del grado de cumplimiento en la publicación de los informes que se demandó en el lineamiento antes enunciado: 


\begin{tabular}{|c|c|c|}
\hline Entidades & $\begin{array}{l}\text { Informes } \\
\text { reportados }\end{array}$ & $\begin{array}{c}\text { Cumplimiento } \\
(\%)\end{array}$ \\
\hline Comisión de Regulación de Energía y Gas & 10 & 100,00 \\
\hline Departamento Administrativo de la Función Pública, DAFP & 10 & 100,00 \\
\hline Departamento Administrativo Nacional de Estadística, DANE & 10 & 100,00 \\
\hline Departamento Nacional de Planeación, DNP & 10 & 100,00 \\
\hline Ministerio de Agricultura y Desarrollo Rural & 10 & 100,00 \\
\hline Ministerio de Comercio, Industria y Turismo & 10 & 100,00 \\
\hline Ministerio de Defensa Nacional & 10 & 100,00 \\
\hline Ministerio de Educación Nacional & 10 & 100,00 \\
\hline Ministerio de Hacienda y Crédito Público & 10 & 100,00 \\
\hline Ministerio de Justicia y del Derecho & 10 & 100,00 \\
\hline Ministerio de Transporte & 10 & 100,00 \\
\hline $\begin{array}{l}\text { Departamento Administrativo de Ciencia, Tecnología e } \\
\text { Innovación, Colciencias }\end{array}$ & 9 & 90,00 \\
\hline Departamento Administrativo para la Prosperidad Social, DPS & 9 & 90,00 \\
\hline Ministerio de Minas y Energía & 9 & 90,00 \\
\hline Ministerio de Trabajo & 9 & 90,00 \\
\hline Presidencia de la República & 9 & 90,00 \\
\hline Ministerio de Ambiente y Desarrollo Sostenible & 8 & 80,00 \\
\hline Ministerio del Interior & 8 & 80,00 \\
\hline Ministerio de Salud y de la Protección Social & 7 & 70,00 \\
\hline Ministerio de Vivienda, Ciudad y Territorio & 7 & 70,00 \\
\hline Superintendencia de Puertos y Transportes & 7 & 70,00 \\
\hline Autoridad Nacional de Licencias Ambientales, ANLA & 6 & 60,00 \\
\hline Ministerio de Cultura & 6 & 60,00 \\
\hline $\begin{array}{l}\text { Ministerio de Tecnologías de la Información y de las } \\
\text { Comunicaciones, MinTIC }\end{array}$ & 6 & 60,00 \\
\hline Parques Nacionales Naturales de Colombia & 6 & 60,00 \\
\hline Comisión de Regulación de Agua Potable y Saneamiento Básico & 5 & 50,00 \\
\hline Comisión de Regulación de Comunicaciones & 5 & 50,00 \\
\hline $\begin{array}{l}\text { Departamento Administrativo del Deporte, la Recreación, } \\
\text { la Actividad Física y el Aprovechamiento del Tiempo Libre, } \\
\text { Coldeportes }\end{array}$ & 5 & 50,00 \\
\hline Ministerio de Relaciones Exteriores & 3 & 30,00 \\
\hline Promedio informes reportados & 8 & 81 \\
\hline
\end{tabular}

Tabla 1

Ranking de entidades de la rama ejecutiva del orden nacional nivel central que cumplieron con la publicación de los informes en su sitio web

Fuente: elaboración propia 
A la fecha de corte para la investigación (31 de enero de 2015), las entidades deberían tener como mínimo 10 informes publicados en su página web: uno por el año 2011 y de 2012 a 2014 tres por año.

De lo anterior, se evidencia que el promedio de informes publicados en el conjunto de las entidades analizadas es de 8 , es decir, un grado de cumplimiento del $81 \%$; este es un nivel medio-alto, lo cual permite rechazar la primera hipótesis planteada, pues no es el 100\% de los informes, como debería ser.

De manera particular, once (11) entidades de las 29 que han publicado informes de control interno en sus sitios web, logran un cumplimiento total en la divulgación de los informes, y representan el 38\% de las entidades; en principio, es un nivel bajo de cumplimiento total, toda vez que el reporte es un mandato legal, por ende, se esperaría que todas las entidades lo hubiesen hecho visible.

No obstante, tres (3) de las cinco (5) entidades que arrojan un cumplimiento del $90 \%$ (9 informes), aparentemente ya publicaron el informe que les haría falta para llegar a los 10 reportes, pero cuando se consulta el documento, la página genera un error. Tal es la suerte del Departamento Administrativo para la Prosperidad Social (en adelante, DPS), el Ministerio de Minas y Energía, y la Presidencia de la República. Otras más son las entidades en las cuales se presenta la generación del error, pero no están cercanas a llegar a los 10 informes (100\% de cumplimiento).

Caso similar le ocurre al Departamento Administrativo de Ciencia, Tecnología e Innovación (en adelante, Colciencias), pues en este caso, el enlace del informe correspondiente al último período de 2014 (12-jul a 12-nov) le asociaron el informe correspondiente al período noviembre de 2012 a marzo de 2013, razón por la cual no obtiene el 100\% de los informes divulgados.

Otro caso particular es el del Ministerio de Cultura, pues se requería una contraseña para poder consultar los informes, razón por la cual no se pudo determinar el cumplimiento de la obligación de reporte sujeto de análisis.

De lo anterior se puede sugerir que si se solucionaran los inconvenientes con la publicación de los informes, se llegaría a un número de entidades que cumplirían en su totalidad con la divulgación de los informes que representan el $50 \%$ de las entidades obligadas y que cuentan con un sitio web.

De las 29 entidades, 14 publican los informes en lugares de difícil acceso para los usuarios, es decir, se deben hacer varios procesos en la página para poder llegar a ellos, lo cual significa una limitación en el acceso a la información y es un aspecto que las entidades deben mejorar, más aún cuando se utilizan las páginas web como medio de divulgación.

\section{Hipótesis de trabajo 2}

Para el desarrollo de esta hipótesis, se evaluó el cumplimiento en la divulgación de los productos mínimos requeridos en los informes, de acuerdo con lo establecido en los tres módulos señalados en el Manual Técnico del MECI, a saber: planeación y gestión, evaluación y seguimiento, e información y comunicación.

En esta medida, se presenta a continuación la evaluación sobre el grado de cumplimiento 
de cada uno de los productos en el conjunto de las entidades abordadas, para poder establecer el grado de cumplimiento de cada una de las entidades que tiene expuesto su informe de control interno pormenorizado a noviembre de 2014, es decir, sobre un total de 25 entidades. Con lo anterior, se elaboró un ranking del $e$ - control por entidades; con ello, se dará respuesta a la segunda hipótesis de trabajo.

En este sentido, se exponen los resultados obtenidos en cuanto al grado de cumplimiento en la divulgación de los productos mínimos por cada uno de los módulos establecidos en el MECI y que fueron evidenciados en los informes publicados.

\begin{tabular}{|c|c|c|c|}
\hline Elemento & Producto mínimo & Puntuación & $\begin{array}{l}\text { Cumplimiento } \\
(\%)\end{array}$ \\
\hline \multicolumn{4}{|c|}{ Componente de talento humano } \\
\hline \multirow{3}{*}{$\begin{array}{l}\text { * Acuerdos, compromisos } \\
\text { o protocolos éticos }\end{array}$} & $\begin{array}{l}\text { Acto administrativo que adopta el documento con } \\
\text { los principios y valores de la entidad }\end{array}$ & 11 & 44,00 \\
\hline & $\begin{array}{l}\text { Documento con los principios y valores de la } \\
\text { entidad }\end{array}$ & 14 & 56,00 \\
\hline & $\begin{array}{l}\text { Estrategias de socialización permanente de los } \\
\text { principios y valores de la entidad }\end{array}$ & 14 & 56,00 \\
\hline \multirow{6}{*}{$\begin{array}{l}\text { * Desarrollo del talento } \\
\text { humano }\end{array}$} & Manual de funciones y competencias laborales & 9 & 36,00 \\
\hline & Plan de incentivos (anual) & 9 & 36,00 \\
\hline & $\begin{array}{l}\text { Plan institucional de formación y capacitación } \\
\text { (anual) }\end{array}$ & 17 & 68,00 \\
\hline & Programa de bienestar (anual) & 17 & 68,00 \\
\hline & Programa de inducción y reinducción & 13 & 52,00 \\
\hline & Sistema de evaluación del desempeño & 10 & 40,00 \\
\hline \multicolumn{4}{|c|}{ Componente de dirección estratégica } \\
\hline \multirow{2}{*}{$\begin{array}{l}* \text { Estructura } \\
\text { organizacional }\end{array}$} & $\begin{array}{l}\text { Estructura organizacional de la entidad que facilite } \\
\text { la gestión por procesos }\end{array}$ & 10 & 40,00 \\
\hline & Manual de funciones y competencias laborales & 9 & 36,00 \\
\hline \multirow{3}{*}{ * Indicadores de gestión } & $\begin{array}{l}\text { Definición de indicadores de eficiencia y } \\
\text { efectividad, que permiten medir y evaluar el } \\
\text { avance en la ejecución de los planes, programas y } \\
\text { proyectos }\end{array}$ & 13 & 52,00 \\
\hline & $\begin{array}{l}\text { Revisión de la pertinencia y utilidad de los } \\
\text { indicadores }\end{array}$ & 14 & 56,00 \\
\hline & Seguimiento de los indicadores & 14 & 56,00 \\
\hline \multirow{3}{*}{$\begin{array}{l}\text { * Modelo de operación } \\
\text { por procesos }\end{array}$} & Divulgación de los procedimientos & 10 & 40,00 \\
\hline & Mapa de procesos & 16 & 64,00 \\
\hline & $\begin{array}{l}\text { Proceso de seguimiento y evaluación que incluya } \\
\text { la evaluación de la satisfacción del cliente y partes } \\
\text { interesadas }\end{array}$ & 9 & 36,00 \\
\hline \multirow{4}{*}{$\begin{array}{l}* \text { Planes, programas y } \\
\text { proyectos }\end{array}$} & $\begin{array}{l}\text { La misión y visión institucionales adoptadas y } \\
\text { divulgadas }\end{array}$ & 7 & 28,00 \\
\hline & Objetivos institucionales & 6 & 24,00 \\
\hline & Planeación & 15 & 60,00 \\
\hline & Planes, programas y proyectos & 19 & 76,00 \\
\hline
\end{tabular}




\begin{tabular}{|c|c|c|c|}
\hline Elemento & Producto mínimo & Puntuación & $\begin{array}{l}\text { Cumplimiento } \\
(\%)\end{array}$ \\
\hline \multirow{2}{*}{ * Políticas de Operación } & $\begin{array}{l}\text { Establecimiento y divulgación de las políticas de } \\
\text { operación }\end{array}$ & 12,5 & 50,00 \\
\hline & $\begin{array}{l}\text { Manual de operaciones o su equivalente adoptado } \\
\text { y divulgado }\end{array}$ & 12 & 48,00 \\
\hline \multicolumn{4}{|c|}{ Componente de administración de riesgos } \\
\hline \multirow{6}{*}{$\begin{array}{l}\text { * Análisis y valoración de } \\
\text { riesgos }\end{array}$} & Análisis del riesgo & 9 & 36,00 \\
\hline & Controles & 8 & 32,00 \\
\hline & Evaluación de controles existentes & 9 & 36,00 \\
\hline & Mapa de riesgos de proceso & 12 & 48,00 \\
\hline & Mapa de riesgos institucional & 11 & 44,00 \\
\hline & Valoración del riesgo & 9 & 36,00 \\
\hline \multirow{2}{*}{$\begin{array}{l}* \text { Identificación de } \\
\text { riesgos }\end{array}$} & $\begin{array}{l}\text { Identificación de los factores internos y externos } \\
\text { de riesgo }\end{array}$ & 10 & 40,00 \\
\hline & $\begin{array}{l}\text { Riesgos identificados por procesos que puedan } \\
\text { afectar el cumplimiento de objetivos de la entidad }\end{array}$ & 9 & 36,00 \\
\hline \multirow{2}{*}{$\begin{array}{l}\text { * Políticas de } \\
\text { administración de riesgos }\end{array}$} & $\begin{array}{l}\text { Definición por parte de la alta dirección de } \\
\text { políticas para el manejo de los riesgos }\end{array}$ & 15 & 60,00 \\
\hline & $\begin{array}{l}\text { Divulgación del mapa de riesgos institucional y } \\
\text { sus políticas }\end{array}$ & 12 & 48,00 \\
\hline
\end{tabular}

Tabla 2

Grado de cumplimiento en la divulgación de los Productos Mínimos correspondientes al Módulo de Planeación y Gestión

Fuente: elaboración propia, a partir del Manual Técnico del Modelo Estándar de Control Interno para el Estado colombiano, MECI 2014 (DAFP, 2014b)

En la tabla 2 se puede observar que los aspectos más divulgados en los informes tienen qué ver con la planeación, el modo de ejecución de los procesos, planes, programas y proyectos, y lo asociado con las capacitaciones que el personal de la entidad debería tener para poder lograr ejecutarlos de manera adecuada. Sin embargo, el grado de divulgación es medio, pues el rango es entre 60 y $76 \%$; con este último porcentaje solo hay uno.

Entidades como el DAFP, el Ministerio del Interior, el Ministerio de Transporte y la Superintendencia de Puertos y Transportes muestran los avances y estadísticas de la ejecución del presupuesto que les fue asignado. Así mismo, el Ministerio de Defensa es una de las pocas entidades que hacen capacitaciones sobre finanzas y generan múltiples guías sobre el tema; también aprovecha las herramientas tecnológicas para dirigir las capacitaciones.

Por otra parte, la divulgación y el establecimiento de políticas, códigos de ética y mapas de riesgos son aspectos con un grado de divulgación medio con rangos entre 40 y $56 \%$. No obstante, algunas entidades que mencionaban estos aspectos lo hicieron para manifestar que aún no tenían la política o no había sido aprobada.

Siguiendo la línea de los manuales, el producto mínimo denominado Manual de funciones $y$ competencias laborales está duplicado pues aparece en el componente de talento humano y en el de estructura organizacional, y tiene los mismos li- 
neamientos de acuerdo con el Manual Técnico del Modelo Estándar de Control Interno para el Estado colombiano, MECI 2014, emitido por el DAFP.

Los resultados también muestran un bajo nivel de cumplimiento en lo que concierne a todo el componente de administración del riesgo, que es vital en todas las entidades, y más en la evaluación de que trata este artículo, ya que por medio de este componente la entidad puede identificar, evaluar y gestionar aquellos eventos negativos, tanto internos como externos, que puedan afectar o impedir el logro de los objetivos institucionales (DAFP, 2014b).

El flagelo de la corrupción fue el motor de la emisión de un Estatuto Anticorrupción; por ende, la inclusión de una evaluación cuatrimestral sobre este tema en las entidades del ejecutivo resulta relevante, sobre todo cuando se espera que las entidades asuman una postura preventiva y, por ello, se requiere que hagan una evaluación de los distintos riesgos que pueden enfrentar.

En términos generales, este módulo es el más importante para las entidades pues de él se desprenden los parámetros para su planeación de gestión y ejecución, con el fin de que alcancen sus objetivos.

De este modo, la tabla 2 muestra un bajo nivel de divulgación en los parámetros establecidos en este módulo, pues de 33 productos mínimos tan solo 6 superan el $60 \%$.

Por otra parte, la mayoría de los informes está reportando únicamente si la entidad cuenta con el producto mínimo, pero no da cuenta de sus avances en el período evaluado, no da las recomendaciones correspondientes ni detalla los aspectos narrados.
En este escenario, sin información relevante sobre los controles asociados con la ejecución del presupuesto, el seguimiento detallado al cumplimiento de los planes, programas y proyectos, y sus respectivos correctivos y/o recomendaciones sobre estos, la sociedad no podría entender los parámetros básicos de la planeación prevista en cada entidad ni reconocer su desarrollo, pues desconoce los avances y medidas sugeridas en el control interno para que la entidad pueda cumplir a cabalidad la gestión y conseguir las metas en el escenario de eficiencia, eficacia y economía previsto para este tipo de instituciones.

Así mismo, como se mencionaba en párrafos anteriores, se advierte sobre la falta de implementación y aplicación de las políticas de administración del riesgo, y las evaluaciones y hallazgos sobre estos, lo que imposibilita el debido seguimiento y control por parte de la ciudadanía para generar alertas y actuaciones que permitan evitar escenarios no deseados de corrupción y/o de prácticas indebidas en la gestión de las entidades.

En cuanto al segundo módulo, se obtuvieron los siguientes resultados (ver tabla 3).

En este módulo, se evidencia la divulgación del programa de auditorías (plan de auditorías) y de las herramientas de autoevaluación, que son los productos mínimos con mayor grado de cumplimiento 92 y 84\%, respectivamente. Esto indica que las entidades se preocupan por hacer seguimientos y mediciones de resultados y procesos, con el fin de determinar las fallas que puedan afectar el cumplimiento de los objetivos institucionales. 


\begin{tabular}{|c|c|c|c|}
\hline Elemento & Producto mínimo & Puntuación & $\begin{array}{c}\text { Cumplimiento } \\
(\%)\end{array}$ \\
\hline \multicolumn{4}{|c|}{ Componente de autoevaluación institucional } \\
\hline \multirow{3}{*}{$\begin{array}{l}\text { * Autoevaluación de } \\
\text { control y gestión }\end{array}$} & Actividades de sensibilización a los servidores sobre la & & \\
\hline & cultura de la autoevaluación & 8 & 32,00 \\
\hline & Herramientas de autoevaluación & 21 & 84,00 \\
\hline \multicolumn{4}{|c|}{ Componente de auditoría interna } \\
\hline \multirow{3}{*}{ * Auditoría interna } & Procedimiento de auditoría interna & 10 & 40,00 \\
\hline & Programa de auditorías - Plan de auditoría & 23 & 92,00 \\
\hline & Informe ejecutivo anual de control interno & 10 & 40,00 \\
\hline \multicolumn{4}{|c|}{ Componente de planes de mejoramiento } \\
\hline \multirow{4}{*}{$\begin{array}{l}\text { * Plan de } \\
\text { mejoramiento }\end{array}$} & Herramientas de evaluación definidas para la & & \\
\hline & elaboración del plan de mejoramiento & 15 & 60,00 \\
\hline & Seguimiento al cumplimiento de las acciones & & \\
\hline & definidas en el plan de mejoramiento & 18 & 72,00 \\
\hline
\end{tabular}

Tabla 3

Grado de cumplimiento en la divulgación de los productos mínimos correspondientes al módulo de evaluación y seguimiento Fuente: elaboración propia, a partir del Manual Técnico del Modelo Estándar de Control Interno para el Estado colombiano, MECI 2014 (DAFP, 2014b)

Lo anterior tiene sentido, toda vez que el componente con mayor grado de divulgación es el de planes de mejoramiento, que muestra avances parciales en todos sus productos mínimos con un porcentaje medio de $66 \%$. En este sentido, las entidades procuran tener acciones que corrijan las dificultades y/o debilidades encontradas en sus procesos de auditoría interna y de autoevaluación, y las de entidades externas (órganos de control) con el fin de fortalecer sus procesos de gestión.

En el lado opuesto está el producto de sensibilización a los empleados respecto a la cultura de autoevaluación, con un grado de divulgación de $32 \%$, lo cual indica que las entidades no toman este factor como herramienta complementaria a las demás, pero este es un instrumento básico en el proceso, ya que son los mismos funcionarios los que pueden evidenciar mayores hallazgos y/o recomendaciones de mejora.
La misma suerte corre el producto de procedimientos de auditoría interna, pues solo el $40 \%$ de las entidades hace mención a la definición de los procedimientos adoptados, que le servirían de herramienta de control permanente en la gestión administrativa.

Sin embargo, la divulgación de este módulo alcanza un 60\% de cumplimiento - el módulo más alto en términos de cumplimiento-, lo que evidencia un interés general por la medición y seguimiento de sus procesos. No obstante, es un porcentaje medio, teniendo en cuenta las mismas aclaraciones del módulo anterior (para la tabla 2), en lo que compete a la limitación del solo cumplimiento, sin mostrar avances y/o recomendaciones.

En este sentido, la falta de referencia clara en los procedimientos adoptados, planes y lineamientos de auditorías de calidad, gestión y cumplimiento, y en los procedimientos de autoevaluación y seguimiento, con sus respecti- 
vos resultados y/o hallazgos obtenidos en estos procesos, imposibilita a los actores del control social un entendimiento amplio y de fácil reconocimiento que le permita verificar el cumplimiento de actividades con fines de prevención, y críticas que lleven a la corrección y/o sanción social (Transparencia por Colombia, 2004), razones por las cuales no es posible determinar la efectiva gestión y/u omisiones que conduzcan al desvío misional de la entidad, con lo cual incumple entonces el precepto de control, como lo enuncia la Corte Constitucional (1999a):

\begin{abstract}
Este control es principalmente axiológico y finalista, pues propende por asegurar que la gestión institucional de todos los órganos del Estado, se oriente hacia la realización de los fines que constituyen su objetivo y, que esta se realice con estricta sujeción a los principios constitucionales que guían el ejercicio de la función pública.
\end{abstract}

En el tercer módulo, se obtienen los siguientes resultados, como se muestra en la tabla 4.

\begin{tabular}{|c|c|c|c|}
\hline Elemento & Producto mínimo & Puntuación & $\begin{array}{c}\text { Cumplimiento } \\
(\%)\end{array}$ \\
\hline \multicolumn{4}{|c|}{ Componente de información y comunicación } \\
\hline \multirow{8}{*}{$\begin{array}{l}\text { * Información } \\
\text { y comunicación } \\
\text { interna y externa }\end{array}$} & Identificación de las fuentes de información externa & 17 & 68,00 \\
\hline & $\begin{array}{l}\text { Fuentes internas de información (manuales, informes, } \\
\text { actas, actos administrativos) sistematizada y de fácil }\end{array}$ & & \\
\hline & acceso & 12 & 48,00 \\
\hline & Rendición anual de cuentas con la intervención de los & & \\
\hline & distintos grupos de interés, veedurías y ciudadanía & 12 & 48,00 \\
\hline & Tablas de retención documental de acuerdo con lo & & \\
\hline & previsto en la normatividad & 13 & 52,00 \\
\hline & Política de comunicaciones & 8 & 32,00 \\
\hline \multirow{8}{*}{$\begin{array}{l}\text { * Sistemas de } \\
\text { información y } \\
\text { comunicación }\end{array}$} & Manejo organizado o sistematizado de la & & \\
\hline & correspondencia & 8 & 32,00 \\
\hline & Manejo organizado o sistematizado de los recursos & & \\
\hline & físicos, humanos, financieros y tecnológicos & 12 & 48,00 \\
\hline & Mecanismos de consulta con distintos grupos de & & \\
\hline & interés para obtener información sobre necesidades y & & \\
\hline & prioridades en la prestación del servicio & 13 & 52,00 \\
\hline & $\begin{array}{l}\text { Medios de acceso a la información con que cuenta la } \\
\text { entidad }\end{array}$ & 13 & 52,00 \\
\hline
\end{tabular}

Tabla 4

Grado de cumplimiento en la divulgación de los productos mínimos correspondientes al módulo de información y comunicación

Fuente: elaboración propia, a partir del Manual Técnico del Modelo Estándar de Control Interno para el Estado colombiano, MECI 2014 (DAFP, 2014b)

De manera particular, nótese cómo de forma vinculante se relacionan en porcentaje la “identificación de las fuentes de información externa" con los "mecanismos de consulta con 
distintos grupos de interés" y los "medios de acceso a la información con que cuenta la entidad" con 62, 52 y $52 \%$, respectivamente, lo cual sugiere que las entidades procuran mantener herramientas de contacto directo con los grupos de interés y de cierto modo les facilitan el acceso a su información y a sus servicios.

No obstante, muy pocas entidades - como el Ministerio de Hacienda, la Presidencia, DAFP y DPS - muestran avances o relatan lo que han trabajado en el período evaluado. Por eso es importante resaltar lo que hacen estas entidades y más el DPS que además de relatar lo realizado durante el período, publica la inversión (salida de dinero) en que incurrió para ello.

Adicionalmente, un producto mínimo (que también debería verse ligado a los mencionados en los párrafos anteriores) - el de manejo organizado o sistematizado de la correspondencia-es el indicador con más bajo porcentaje de participación, 32\%, lo cual permite interpretar que a pesar de que las entidades hacen un esfuerzo por tener contacto con la ciudadanía, aún no cuentan con directrices claras para este manejo documental ni para manejar la correspondencia recibida y generar respuestas a los grupos de interés (DAFP, 2014b).

De igual forma, como se advertía en la tabla 2, las entidades hablan en un nivel medio-bajo de las políticas que adoptan para el funcionamiento y gestión en la entidad, y en este componente se observa el mismo comportamiento en el producto mínimo de "políticas de comunicación", que solo en 8 ocasiones (32\%) fueron divulgadas.

Ya en términos generales, en la tabla 4, todos los productos mínimos están en un grado medio y logran en promedio un cumplimiento de $48 \%$, lo cual denota un bajo porcentaje de divulgación teniendo en cuenta que este módulo es un eje transversal, es decir, hace parte de todas las actividades de control que se veían en las tablas anteriores.

Hubo entidades que en los informes hablaban del Manual 3.1 para la implementación de la estrategia de gobierno en línea para entidades del orden nacional, interpretando con eso que ya tenían identificados los aspectos mínimos a publicar de acuerdo con ese manual y que, con ello, se satisface lo exigido en el componente de información y comunicación, lo que puede ser cierto pero en la mayoría de casos no describe en qué términos se viene cumpliendo con esta estrategia. Por ello, la calificación dada en ese aspecto fue de cero (0), toda vez que si bien el Manual 3.1 para la implementación de la estrategia de gobierno en línea para entidades del orden nacional es un aspecto de cumplimiento y tiene relación con el componente de información y comunicación, el Manual Técnico del Modelo Estándar de Control Interno para el Estado colombiano, MECI 2014 (DAFP, 2014b) no menciona de forma taxativa la divulgación obligatoria de los lineamientos orientados en el Manual 3.1 para la implementación de la estrategia de gobierno en línea para entidades del orden nacional.

Así las cosas, la falta de información sobre los resultados de los controles asociados con los proyectos, obras, contratos y administración de los recursos le impide a la ciudadanía verificar su estado y avances para el debido seguimiento y control, lo que facilita omisiones, desvíos de recursos por la ausencia de visibilidad y de difusión de información oficial. En este mismo sentido, impide identificar las entidades y/o personas 
que están desarrollando las obras y contratos, lo que imposibilita la identificación de la concentración de recursos en manos de un mismo tercero y genera riesgos de corrupción.

De este mismo modo, la falta de divulgación y de estrategias de comunicación, y el no establecimiento de mecanismos para la interacción con el público y personal de la misma entidad imposibilitan la comunicación directa con estos e impiden la administración electrónica como un método para mejorar la transparencia infor- mativa, la cual ayudaría a mejorar la prestación de los servicios públicos y, a su vez, la participación de los ciudadanos en la gestión pública, sin ofrecer nuevas formas de rendición de cuentas (Alcaide, Rodríguez \& Garde, 2014).

Con los resultados obtenidos en los tres módulos, se logra la nota global para cada una de las entidades, con lo que se puede establecer el ranking de $e$-control para las entidades del Estado pertenecientes a la rama ejecutiva del orden nacional nivel central.

\begin{tabular}{|c|c|c|}
\hline Entidades & $\begin{array}{l}\text { Puntaje } \\
\text { obtenido }\end{array}$ & $\begin{array}{c}\text { Cumplimiento } \\
(\%)\end{array}$ \\
\hline Departamento Administrativo para la Prosperidad Social, DPS & 48 & 97,96 \\
\hline Ministerio de Agricultura y Desarrollo Rural & 43 & 87,76 \\
\hline Ministerio de Minas y Energía & 41 & 83,67 \\
\hline Presidencia de la República & 40 & 81,63 \\
\hline Ministerio de Justicia y del Derecho & 39 & 79,59 \\
\hline Autoridad Nacional de Licencias Ambientales, ANLA & 36 & 73,47 \\
\hline Comisión de Regulación de Energía y Gas & 29 & 59,18 \\
\hline Departamento Administrativo de la Función Pública, DAFP & 28 & 57,14 \\
\hline Ministerio de Vivienda, Ciudad y Territorio & 28 & 57,14 \\
\hline Parques Nacionales Naturales de Colombia & 28 & 57,14 \\
\hline Ministerio de Ambiente y Desarrollo Sostenible & 27 & 55,10 \\
\hline Ministerio de Educación Nacional & 27 & 55,10 \\
\hline Ministerio de Hacienda y Crédito Público & 26 & 53,06 \\
\hline Ministerio de Trabajo & 26 & 53,06 \\
\hline Superintendencia de Puertos y Transportes & 26 & 53,06 \\
\hline Ministerio de Transporte & 20 & 40,82 \\
\hline Ministerio de Comercio, Industria y Turismo & 15 & 30,61 \\
\hline Ministerio de Relaciones Exteriores & 15 & 30,61 \\
\hline Ministerio del Interior & 15 & 30,61 \\
\hline \multicolumn{3}{|l|}{ Departamento Administrativo del Deporte, la Recreación, la Actividad } \\
\hline Física y el Aprovechamiento del Tiempo Libre, Coldeportes & 13,5 & 27,55 \\
\hline Departamento Administrativo Nacional de Estadística, DANE & 12 & 24,49 \\
\hline Ministerio de Defensa Nacional & 8 & 16,33 \\
\hline Departamento Nacional de Planeación, DNP & 5 & 10,20 \\
\hline Comisión de Regulación de Agua Potable y Saneamiento Básico & 2 & 4,08 \\
\hline Ministerio de Tecnologías de la Información y de las Comunicaciones & 0 & 0,00 \\
\hline Promedio informes reportados & 23,9 & 48,78 \\
\hline
\end{tabular}

Tabla 5

Ranking del e-control en entidades de la rama ejecutiva del orden nacional nivel central que dan cumplimiento a la divulgación de los productos mínimos establecidos en el MECI 2014

Fuente: elaboración propia 
La entidad con el mejor puntaje (grado de cumplimiento) fue el DPS, pues de 49 puntos alcanzó 48, es decir, un grado de cumplimiento del 97,9\% de la información mínima a mencionar en el informe pormenorizado sobre el estado del control interno de la entidad. Esta entidad cumplió el 97\% del módulo de planeación y gestión; y el 100\% en los módulos de evaluación y seguimiento, e información y comunicación. Sin embargo, por más de que ocupe el primer lugar y sea un informe organizado, le falta mejorar la presentación en lo que compete a los lineamientos dados por el DAFP, ya que de manera parcial se limita a mencionar el mero cumplimiento de establecer los productos mínimos, y no habla sobre los avances, que es como debería mostrarse el informe.

En contraste, el Ministerio de Tecnologías de la Información y de las Comunicaciones ocupa el último lugar, con un grado de cumplimiento cero (0), es decir, el informe no menciona ningún producto mínimo requerido. Esto se debe a que el informe se centró en divulgar el estado de cumplimiento en la implementación del nuevo MECI, y cómo lo ha hecho en cada etapa, sin hablar qué está haciendo en cada producto mínimo.

Un caso similar es el de la Comisión de Agua Potable y Saneamiento Básico, pues también es un informe atípico, que no cumple los requerimientos del Modelo de informe ni el Manual Técnico del MECI, emitidos por el DAFP. Por ello, su grado de cumplimiento es del 4,08\%, pues dos productos mínimos fueron mencionados. Si bien en cierta medida es entendible la situación que se comenta, para el control social se requiere que no solo se infor- me sobre el estado de implementación o de ajuste con los nuevos lineamientos, sino que se informe de cada uno de los productos mínimos, dado que durante ese tiempo la entidad sigue en funcionamiento $y$, por ende, puede estar expuesta a prácticas que no privilegien el cumplimiento de sus objetivos misionales y financieros.

El mismo caso de informes atípicos ocurre en Coldeportes y el Ministerio de Minas; sin embargo, de estos informes se pudo evidenciar que daban cuenta de los productos mínimos, motivo por el cual su puntuación no fue tan baja $(13,5,27,5 \%$ y $41,83,6 \%)$ como la de las entidades anteriormente mencionadas.

De igual forma, los resultados expuestos en la tabla 5 muestran que las entidades mencionan en promedio el 48,78\% de los productos mínimos requeridos en el informe. Este es un nivel medio-bajo, teniendo en cuenta que, como se ha venido advirtiendo a lo largo de los análisis, la mayoría de las entidades se limita a mencionar expresamente lo establecido en el Manual Técnico del Modelo Estándar de Control Interno para el Estado colombiano, MECI 2014, respecto a los productos mínimos, y no da cuenta de los avances que se hacen en el período de evaluación, como también lo pide el Modelo de Informe, emitido por el DAFP.

Sin embargo, hay que dar mención a ocho entidades, pues cumplen en un $90 \%$ lo que se esperaría ver en los informes, es decir, la estructuración de avances, debilidades y recomendaciones: DAFP, Ministerio de Justicia, Ministerio de Trabajo, Ministerio de Transporte, Ministerio de Vivienda, Parques Nacionales Naturales, Superintendencia de Puertos y 
Transporte, y la Presidencia de la República. De esta última, hay que resaltar que es uno de los mejores informes, aunque su reporte no lo hacen bajo el MECI 2014 (Decreto 943 del 21 de mayo de 2014), sino con los parámetros de la reglamentación anterior.

Por último, aun cuando las entidades estén divulgando la información requerida en un $48,78 \%$, la gran mayoría de las entidades ya adoptó el nuevo esquema del MECI 2014 (15 entidades aproximadamente), y reconocen en algunos casos, que están en un 50\% de grado de avance de implementación del nuevo MECI, incluso, algunas entidades van hasta ahora comenzando la adaptación. La misma suerte corren las otras 10 entidades evaluadas que reportaron bajo el esquema de la reglamentación anterior (Decreto 1599 de 2005).

De acuerdo con el Decreto 943 de 2014, las entidades que fueron creadas antes de 2012, tenían un plazo de 7 meses para adoptar el nuevo modelo, plazo que vencía el 21 de diciembre de 2014. Los informes en estudio fueron emitidos en noviembre y diciembre, razón por la cual las entidades debieron dar cuenta de la culminación de este proceso.

Por lo expuesto, en los análisis sobre los distintos módulos y en el ranking se hace evidente que las entidades del Estado pertenecientes a la rama ejecutiva del orden nacional nivel central no cumplen con el 100\% de los productos mínimos, por lo que la hipótesis número dos se rechaza por completo. Esto limita el acceso a la información relevante para realizar el control social.

El atraso en la adaptación al nuevo MECI impide uno de los propósitos generales de su actualización, el cual permitiría aclarar conceptos, que facilitarían su comprensión y, a la vez, pretende introducir estrategias de simplificación que hagan efectiva su operación (DAFP, 2014b). Por tal motivo, la falta de publicación efectiva de los productos mínimos que presenten los hallazgos, avances, recomendaciones y/o debilidades, y la ausencia de claridad en los informes y el no reconocimiento de nuevos lineamientos para la debida gestión, impiden a la ciudadanía entender, identificar y realizar un seguimiento de verificación a las actuaciones y ejecución administrativa que desarrollan las entidades, sin poder entonces llevar a cabo el propósito esencial del control social, el cual va desde la prevención a la acusación y sanción, concebidas por el reconocimiento oportuno y transparente de la información, el seguimiento al cumplimiento de compromisos y metas de las entidades (Transparencia por Colombia, 2004), manifestar y/o alertar sobre posibles riesgos de la gestión pública (Ministerio de Interior y de Justicia, 2006).

\section{Conclusiones}

Los escándalos sobre corrupción están haciendo mella en el crecimiento socioeconómico de los países, y cada vez son más recurrentes; cuando se piensa que ya se ha tocado fondo, surgen nuevas modalidades y escándalos. En un país como Colombia, cuya economía es terciaria, y en donde se busca avanzar y estar a la par con países de primer nivel, este flagelo es de mayor impacto que en países que tienen un soporte socioeconómico más avanzado. Por esto, surgen múltiples acuerdos internacionales, 
propuestas de cooperación, códigos de buen gobierno e iniciativas como la que fue objeto de estudio en este artículo, los cuales en su conjunto se espera contribuyan en la mitigación de los riesgos de corrupción.

Para que este proceso sea efectivo, se debe involucrar a la ciudadanía y educar a todo el Estado, concienciándolos no solo en materia ética, sino también de participación en el control mediante veedurías o haciéndose partícipes en escenarios que posibilitan la rendición de cuentas y la transparencia de las entidades. Así mismo, los gestores y/o actores públicos deben hacer que estas prácticas sean posibles y coadyuvar al cumplimiento de los mandatos legales existentes y, por qué no, generando nuevas herramientas de transparencia y participación.

Los informes pormenorizados de control interno son una herramienta importante para ejercer el control ciudadano y llevar a cabo evaluaciones sobre las actuaciones de los gestores públicos en cada entidad, dado que sirven en cierta medida, para realizar un monitoreo a las entidades y a sus decisiones.

Con el propósito de evidenciar la efectividad en la divulgación cuatrimestral de los informes pormenorizados en los sitios web, como herramienta de control y seguimiento en las instituciones públicas colombianas de carácter ejecutivo, la presente investigación evaluó el grado de cumplimiento en la divulgación de estos por parte de las entidades públicas de la rama ejecutiva del orden nacional nivel central, para dar cumplimiento a lo establecido en la Ley 1474 de 2011. Así mismo, se analizó el contenido de los aspectos expuestos en estos infor- mes, siguiendo los parámetros previstos en el Manual Técnico del Modelo Estándar de Control Interno para el Estado colombiano, MECI 2014 y el Modelo de informe propuesto por el DAFP para este fin.

Como conclusión, estas instituciones evidencian un grado medio-alto de divulgación de los informes en sus páginas web, lo cual posibilita en cierta medida los propósitos esenciales del reporte por parte del responsable de control interno, es decir, poder realizar un permanente monitoreo a la gestión de las entidades y presentar los resultados ante todas las partes interesadas para que se ejerza el control correspondiente. Sin embargo, como se advirtió en el análisis de la tabla 1, las entidades no cumplen en un $100 \%$ esta obligación y es imperativo hacerlo, so pena de las sanciones disciplinarias por omisión a este mandato.

De igual forma, se evidenció un nivel medio-bajo en el cumplimiento de la información mínima a reportar, tanto en términos de fondo como de forma, pues las entidades se limitan al mero cumplimiento formal de decir si tienen o no el producto mínimo, pero no muestran hallazgos, avances y/o estrategias de mejora.

Por último, con los resultados obtenidos se advierte la necesidad de monitorear y generar controles por parte de los entes reguladores, con el fin de garantizar el cumplimiento en la publicación de los informes y el contenido que allí se debe consignar, y así lograr mejores herramientas efectivas de control y monitoreo que cooperen en la reducción de riesgos de corrupción y, por otro lado, una comunicación con las partes interesadas. Esto también se puede lograr si se vincula en la estrategia de gobierno en línea 
(e-government), lo referente al acceso del informe pormenorizado de control interno, como una práctica de e-control.

\section{Referencias}

Alcaide-Muñoz, Laura; Rodríguez-Bolívar, Manuel Pedro \& Garde-Sánchez, Raquel (2014). Estudio cienciométrico de la investigación en transparencia informativa, participación ciudadana y prestación de servicios públicos mediante la implementación del e-Gobierno. Revista de Contabilidad, 17 (2), 130-142. Disponible en: http:// apps.elsevier.es/watermark/ctl_servlet?_ $\mathrm{f}=10$ \&pident_articulo=90355717\&pident_ usuario $=0 \&$ pcontactid $=$ \&pident_revista $=3$ $68 \&$ ty $=1 \&$ accion $=$ L\&origen $=$ zonadelectur $\mathrm{a} \& w e b=$ www.elsevier.es\&lan $=$ es\&fichero $=$ 368v17n02a90355717pdf001.pdf

Católico, Diego; Gómez, Paola \& Gómez, Angy (2014). Revelación on-line de la información financiera y no financiera de las entidades de la rama ejecutiva del orden nacional en Colombia. Cuadernos de Contabilidad, 15 (37), 77-107. Disponible en: http://revistas.javeriana.edu.co/index.php/ cuacont/article/view/9003/7296

Colombia (1991). Constitución Política, segunda edición corregida. Gaceta Constitucional, 116. Disponible en: http://www.secretariasenado.gov.co/senado/basedoc/constitucion_politica_1991.html

Colombia (2005). Decreto 1599 de 2005, por el cual se adopta el Modelo Estándar de Control Interno para el Estado colombiano. Diario Oficial, 45.920, 26 de mayo de 2005. Disponible en: http://www.alcaldiabogota.
gov.co/sisjur/normas/Norma1. jsp?i=16547\#0

Colombia (2008). Decreto 1151 de 2008, por el cual se establecen los lineamientos generales de la Estrategia de Gobierno en Línea de la República de Colombia, se reglamenta parcialmente la Ley 962 de 2005, y se dictan otras disposiciones. Diario Oficial, 46.960, 14 de abril de 2008. Disponible en: http://www. alcaldiabogota.gov.co/sisjur/normas/ Norma1.jsp?i=29774

Colombia (2012). Decreto 2693 de 2012, por el cual se establecen los lineamientos generales de la Estrategia de Gobierno en Línea de la República de Colombia, se reglamentan parcialmente las Leyes 1341 de 2009 y 1450 de 2011, y se dictan otras disposiciones. Diario Oficial, 48.651, 21 de diciembre de 2012. Disponible en: http://www.alcaldiabogota.gov.co/sisjur/normas/Norma1. jsp?i=51198

Colombia (1993). Ley 87, por la cual se establecen normas para el ejercicio del control interno en las entidades y organismos del Estado y se dictan otras disposiciones. Diario Oficial, 41.120, 29 de noviembre de 1993. Disponible en: http://www. secretariasenado.gov.co/senado/basedoc/ ley_0087_1993.html

Colombia (2011). Ley 1474, por la cual se dictan normas orientadas a fortalecer los mecanismos de prevención, investigación y sanción de actos de corrupción y la efectividad del control de la gestión pública, Estatuto Anticorrupción. Diario Oficial, 48.128, 12 de julio de 2011. Disponible en: http:// 
www.secretariasenado.gov.co/senado/basedoc/ley_1474_2011.html

Colombia (2014). Ley 1712, por medio de la cual se crea la Ley de transparencia y del derecho de acceso a la información pública nacional y se dictan otras disposiciones. Diario Oficial, 49.084, 6 de marzo de 2014. Disponible en: http://www.secretariasenado.gov.co/senado/basedoc/ley_1712_2014. html

Colombia, Corte Constitucional (1999a). Sentencia C-506-99, 14 de julio de 1999, carrera administrativa; empleados de libre nombramiento y remoción, magistrado ponente Fabio Morón-Díaz. Disponible en: http://www.corteconstitucional.gov.co/ relatoria/1999/C-506-99.htm

Colombia, Corte Constitucional (1999b). Sentencia C-957-99, 1 de diciembre de 1999, principio de publicidad; principio de publicidad en materia legislativa, vigencia de la Ley; acto administrativo; prevalencia del derecho sustancial, magistrado ponente Álvaro Tafur-Galvis. Disponible en: http://www.corteconstitucional.gov.co/ relatoria/1999/c-957-99.htm

Colombia, Corte Constitucional (2013). Sentencia C-274-13, 9 de mayo de 2013, revisión integral del proyecto de ley estatutaria por el cual se crea la Ley de Transparencia y del derecho de acceso a la información pública nacional. Delimitación del derecho de acceder a los documentos públicos, en el marco constitucional colombiano, magistrada ponente María Victoria Calle-Correa. Disponible en: http://www.corteconstitucional.gov.co/relatoria/2013/c-274-13.htm
Colombia, Departamento Administrativo de la Función Pública, DAFP (2014a). Decreto 943, por el cual se actualiza el Modelo Estándar de Control Interno, MECI. Diario Oficial, 49.158, 21 de mayo de 2014. Disponible en: http://www.alcaldiabogota. gov.co/sisjur/normas/Norma1.jsp?i=59048

Colombia, Departamento Administrativo de la Función Pública, DAFP (2014b). Manual Técnico del Modelo Estándar de Control Interno para el Estado colombiano, MECI 2014. Disponible en: http://portal. dafp.gov.co/form/formularios.retrive_ publicaciones? $\mathrm{no}=2162$

Colombia, Departamento Administrativo de la Función Pública, DAFP, Dirección de Políticas de Control Interno y Racionalización de Trámites (2008). Manual de implementación, Modelo Estándar de Control Interno para el Estado colombiano, MECI 1000:2005. Disponible en: http:// portal.dafp.gov.co/form/formularios. retrive_publicaciones? $\mathrm{no}=579$

Colombia, Departamento Administrativo de la Función Pública, DAFP \& Instituto de Auditores Internos de Colombia (2013). Guía de auditoría para entidades públicas. Disponible en: http://portal.dafp.gov.co/mod/retrive_ docu_noti?no=186

Colombia, Ministerio de Tecnologías de la Información y las Comunicaciones, MinTIC (2013). Manual 3.1 para la implementación de la estrategia de gobierno en línea para entidades del orden nacional. Disponible en: http://programa.gobiernoenlinea.gov.co/ apc-aa-files/eb0df10529195223c011ca6762bfe39e/manual-3.1.pdf 
Colombia, Ministerio del Interior y de Justicia (2006). Plan nacional de formación para el control social a la gestión pública: mecanismos jurídicos para el control social, momento de fundamentación, Módulo 2. Disponible en: https://issuu.com/veedurianuestrosbarrios/ docs/2_mecanismos_juridicos-para_el_cont

Colombia, Presidencia de la República (2000). Directiva presidencial 002. Gobierno en línea. Bogotá. Disponible en: http://www. alcaldiabogota.gov.co/sisjur/normas/ Norma1.jsp?i=6399

Colombia, Presidencia de la República (2002). Directiva presidencial 12. Lucha contra la corrupción en la contratación estatal. Bogotá. Disponible en: https://www.indumil.gov. co/doc/normas\%20control\%20interno/ Directiva\%20Presidencial/12_2002.pdf Committee of Sponsoring Organizations of the Treadway Commission, COSO (2013). Internal Control - Integrated Framework. Disponible en: http://www. coso.org/documents/990025P_Executive_ Summary_final_may20_e.pdf

Consejo Nacional de Política Económica y Social, CONPES (2010a). Documento 3650. Importancia estratégica de la estrategia en línea. Bogotá, Departamento Nacional de Planeación. Disponible en: https:// colombiadigital.net/images/stories/ Estadisticas\%20INFOTIC/Conpes/ Conpes_3650_2010.pdf

Consejo Nacional de Política Económica y Social, CONPES (2010b). Documento 3654. Política de rendición de cuentas de la rama ejecutiva a los ciudadanos. Bogotá, Departamento Nacional de Planeación. Disponible en: http://portal.dafp.gov.co/form/formularios.retrive_publicaciones?no $=681$

López-Hernández, Antonio Manuel \& Cabeza del Salvador, Ignacio (2002). Control y auditoría en las entidades públicas. Harvard Deusto Finanzas \& Contabilidad, 50, 34-43. Márquez-Gómez, Daniel (2010). Función jurídica de control de la administración pública. México: Instituto de Investigaciones Jurídicas, Universidad Nacional Autónoma de México.

Montilla-Galvis, Omar de Jesús; Montes-Salazar, Carlos Alberto \& Mejía-Soto, Eutimio (2007). Análisis de la fundamentación del Modelo Estándar de Control Interno, MECI I000:2005. Estudios Gerenciales, 23 (104), 47-75. Disponible en: http://www.scielo.org. co/pdf/eg/v23n104/v23n104a03.pdf

Oficina de Naciones Unidas contra la Droga y el Delito, UNODC (2015). Áreas de actuación, Corrupción. UNODC y Corrupción. United Nations Office on Drugs and Crime, Oficina de Enlace y Partenariado en Brasil. Disponible en: http://www.unodc.org/lpobrazil/es/corrupcao/index.html

Pucheta-Martínez, María Consuelo \& CortésFleixas, Josep Lluís (2005). Los informes de auditoría externa de las administraciones públicas. Partida Doble, 163, 96-116.

Rodríguez-Raga, Juan Carlos \& Seligson, Mitchell A. (2011). Cultura política de la democracia en Colombia, 2011. Disponible en: http://www.vanderbilt.edu/lapop/ colombia/2011-Colombia-Cultura-politicade-la-democracia.pdf

Transparencia Internacional (2014). Índice de percepción de la corrupción 2014: el cre- 
cimiento transparente en riesgo. Disponible en: http://www.transparency.org/cpi2014/ results

Transparencia por Colombia (2004). Cuaderno de Transparencia No. 8. El control social a la administración pública en Colombia - Aproximaciones.

- Fecha de recepción: 14 de julio de 2015

- Fecha de aceptación: 14 de marzo de 2016

- Disponible en línea: 15 de mayo de 2016

\section{Para citar este artículo}

Gutiérrez-Ruiz, R. L. \& Católico-Segura, D. F. (2015). E-control en las entidades de la rama ejecutiva del orden nacional en Colombia. Cuadernos de Contabilidad, 16(42), 553-578. http://dx.doi. org/10.11144/Javeriana.cc16-42.eere 